\title{
Evaluation of a Rapid Immunochromatographic test kit to the Gold Standard Fluorescent Antibody test for diagnosis of rabies in animals in Bhutan
}

Tenzin Tenzin ( $\nabla$ tenzinvp@gmail.com )

National Centre for Animal Health

Kelzang Lhamo

$\mathrm{NCAH}$

Purna B Rai

$\mathrm{NCAH}$

Dawa Tshering

$\mathrm{NCAH}$

Pema Gyamtsho

DVH, Trashigang

Jamyang Namgyal

DVH Trashigang

Thrinang Wangdi

RLDC Kanglung

Sangay Letho

RLDC Kanglung

Tuku Rai

SVL Phuentsholing

Sonam Jamtsho

SVL Phuentsholing

Chendu Dorji

RLDC Tshimasham

Sangay Rinchen

RLDC Tshimasham

Lungten Lungten

SVL Deothang

Karma Wangmo

DVH Samtse

Lungten Lungten

DVH Samtse

Pema Wangchuk 
RLDC Zhemgang

\section{Tshewang Gempo}

SVL Gelephu

\section{Kezang Jigme}

SVL Gelephu

Karma Phuntshok

SVL Nganlam

Tenzin Tenzinla

NCAH, Serbithang

Ratna B Gurung

NCAH Serbithang

Kinzang Dukpa

NCAH Serbithang

\section{Research article}

Keywords: rabies virus, diagnosis test, fluorescent antibody test, rapid anigen test, rapid immunochromatographic test, Bhutan

Posted Date: December 20th, 2019

DOl: https://doi.org/10.21203/rs.2.19414/v1

License: (c) (i) This work is licensed under a Creative Commons Attribution 4.0 International License. Read Full License

Version of Record: A version of this preprint was published at BMC Veterinary Research on June 8th, 2020. See the published version at https://doi.org/10.1186/s12917-020-02405-4. 


\section{Abstract}

Background: Rabies kills approximately 59,000 people in the world each year worldwide. Rapid and accurate diagnosis of rabies is important for instituting rapid containment measures and for advising the exposed people for postexposure treatment. The application of a rapid diagnostic tests in the field can greatly enhance disease surveillance activities, especially in resource poor settings.

Methods: From 2012 to 2017, a total of 179 brain tissue samples collected from different animal species (113 dogs, 50 cattle, 10 cats, 3 goats, 2 horses, and 1 bear) suspected of having died due to rabies were selected and tested using the rapid immunochromatographic kit from BioNote $\odot$ company and compared to the Gold Standard Fluorescent Antibody test (FAT) for diagnosis of rabies.

Results: Among 179 samples examined in this study, there was concordance in results by the rapid test and FAT in 115 positive samples and 54 negative samples. Test result were discordant in 10 samples which were positive by FAT, but negative (false negative) by rapid kit. The rapid test kit showed a sensitivity of $92 \%(95 \% \mathrm{Cl}: 85.9-95.6)$ and specificity of $100 \%(95 \% \mathrm{Cl}: 93.4-100)$ using FAT as the gold standard. The positive and negative predicative values were found to be 100\% (95\% Cl:96.7 - 100) and $84 \%$ (95\% Cl: 73.6 - 91.3), respectively. Overall there was $94.41 \%(95 \% \mathrm{Cl}: 90-96.9)$ test agreement (almost perfect agreement) between rapid test and FAT (Kappa value $=0.874$ ).

Conclusions: Our results demonstrate the potential value of the rapid test kit for countries with limited diagnostic resources, including Bhutan. The rapid kit's inability to correctly detect 10 FAT-positive samples (10 out of 179 (5.6\%) were false negatives) in our study could have been due to the low viral load in the samples (<102.0LD50/0.03ml) which could not be detected by the rapid kit as compared with the FAT. The human factor related to the varying experiences of the technicians who performed the test in the field also may have influenced the test result. The rapid test kit is inexpensive, rapid and easy to use in the field or in laboratory setting without the need for special training and can support to enhance rabies surveillance in resource poor countries.

\section{Background}

Rabies is a 100 percent fatal zoonotic disease caused by Lyssavirus of the family Rhabdoviridae and transmitted mainly through the bite of a rabid animal. However, rabies is preventable through three proven and effective interventions - awareness education (i.e. making people aware of how to avoid the bites of rabid dogs and to seek treatment when bitten), mass dog vaccination (i.e. stopping the transmission of rabies at its source when a sufficiently high proportion of the animal reservoir population is immunized), and post-exposure prophylaxis (PEP) (i.e. vaccination/treatment of exposed human victims). Despite the availability of these tools, around 59,000 people die from the disease each year worldwide [1]. Approximately $80 \%$ of human cases occur in rural areas of economically disadvantaged countries in Africa and Asia, and over $40 \%$ of rabies deaths occur in children aged less than 15 years. Dogs are responsible for up to $99 \%$ of human rabies cases in the world. Rapid and accurate diagnosis of 
rabies following exposure to a rabies suspects animal is important for instituting rapid containment measures and for advising the exposed people for postexposure treatment.

In Bhutan, rabies in animals are prevalent mostly in the southern parts of the country that shares border with India. Sporadic outbreaks are also reported in the interior rabies-free areas, especially in eastern Bhutan as a result of incursions and spread from the border towns [2-4]. Between 2006 and 2016, 17 humans have died of rabies in Bhutan, the majority being children below 15 years of age and around 7000 dog bite cases (1026 bites per 100,000 people annually) in humans are reported every year in the country [5-7]. Nevertheless, Bhutan is on the right track to achieve zero human deaths from dogmediated rabies by 2030 as several control measures are in place in line with the global strategic plan of "Zero by $30 "[4,8]$.

The surveillance of rabies depends on investigations conducted on animals showing clinical signs suggestive of rabies, history of exposure and on laboratory investigations of brain tissue samples from carcasses. In Bhutan, the rabies suspect cases in animals are diagnosed based on clinical signs and confirmed by testing brain tissue samples using immunochromatographic test common called as rapid anigen antigen detection test kit (BioNote, South Korea) in the field. In addition, brain samples, irrespective of their rapid test results, are sent to the national veterinary laboratory located at the National Centre for Animal Health in Thimphu for confirmatory testing using the fluorescent antibody test (FAT), the gold standard for rabies diagnosis [9]. Since 2012, the rapid kit has been widely used in Bhutan for field diagnosis of rabies and has enhanced rabies surveillance in the country $[4,10]$. Since it is expensive to set up FAT or molecular diagnostic facilities in all rabies endemic areas in the country, a rapid, accurate, inexpensive and easy to perform test is necessary to diagnose rabies in the field so that control measures can be taken immediately in animals and also in humans exposed to suspects cases in the field.

In this study, we evaluated the test sensitivity, specificity, positive predictive value (PPV) and negative predictive value (NPV) of a rapid antigen detection test kit produced by BioNote, South Korea to a gold standard FAT for diagnosis of rabies virus in various species of domestic animals in Bhutan.

\section{Methods}

\section{Sample source}

A total of 179 brain tissue samples collected from different species of animals ( 113 dogs, 50 cattle, 10 cats, 3 goats, 2 horses, and 1 bear) suspected for rabies in different areas (8 Dzongkhags) (Table 1) at different time points (from 2012 - 2017) (Table 2) in the country were used in this study.

\section{Rapid immunochromatographic test (Anigen Rapid Ag test)}

The Anigen Rapid Rabies Ag Test Kit (BioNote, South Korea) is a chromatographic immunoassay (lateral flow assay) for the qualitative detection of Rabies virus antigen in canine, bovine, raccoon dog's 
secretions of saliva, and brain homogenates. The basic principle behind this test is the fluid migration of a sample along a nitrocellulose membrane. Gold conjugated antibodies bind to antigen in the sample and the antigen-antibody complex is then immobilized by a second antibody which is fixed on the test strip. The rapid test kit has a letter of " $\mathrm{T}$ " and " $\mathrm{C}$ " as test line and control line on the surface of the device. Both the test line and control line in result window are not visible before applying any samples. The control line is used for procedural control. Control line should be always appeared if the test procedure is performed properly and the test reagents of control line are working. A purple test line will be visible in the result window if there are enough rabies virus antigen in the specimen. The specially selected rabies virus antibodies are used in test band as both capture and detector materials.

For this study, the test was performed immediately after harvesting the fresh brain tissue samples in the field according to the manufacturer's instruction (BioNote, Inc). Briefly, the cotton swab supplied along with the kit was used to swab the brain tissue (hippocampus in case of dog and cerebellum in case of cattle, goat, pig, and sheep) and then dipped the swab into the specimen tube containing $1 \mathrm{ml}$ of assay diluent, and stirred/mixed well to ensure a good sample extraction. The swabbing of the brain tissue with swab and mixing into the assay diluent was repeated for 3-5 times to ensure a good sample extraction. The test cassette/device was removed from the foil pouch and placed it horizontally on a flat and dry surface. Using the disposable dropper provided with the kit, four drops of the extracted sample $(100 \mu \mathrm{L})$ was added into the sample hole in the cassette and the result was interpreted within 5-10 minutes according to the manufacturer's instruction. As the test begin to work, the appearance of two colour bands - one on the control line (C) and the other on test line (T) within the result window, no matter which band appears first indicates a positive result. The appearance of only one colour band within the result window indicates a negative result and if the purple color band is not visible within the result window after performing the test, the result is considered invalid. The directions may not have been followed correctly or the test may have deteriorated in which case, as re-test was conducted. The rapid test was conducted in the field by a trained veterinary laboratory technician or by a veterinarian immediately after the suspected rabid animals have been either died, killed or euthanized. The brain tissues samples from non-rabies endemic areas were also collected and tested for rabies virus as part of the surveillance programme. After performing the rapid test in the field, the remaining brain tissue samples were collected and preserved in phosphate glycerol saline (50\%) and shipped to national referral laboratory, (NCAH), Thimphu for confirmatory diagnosis using FAT and to archive for molecular characterization. Although most of the rapid test were conducted in the field using fresh brain tissue samples, some were conducted at the national laboratory using preserved samples.

\section{Fluorescent antibody test}

The FAT was conducted immediately or within one day after receiving the samples from the field to confirm the diagnosis and was performed according to the procedure described by OIE [9]. Briefly, the brain tissue preserved in $50 \%$ glycerol saline were washed with buffered saline to remove the glycerol. The brain tissue impression smears were prepared, air dried and were fixed in chilled acetone for 15-30 minutes. The impression smear slides were air dried and $150 \mu$ l of fluorescein isothiocyanate (FITC) 
conjugate anti-rabies antibody (LIGHT DIAGNOSTICS Rabies DFA Reagent, USA) which was prepared by diluting at 1:20 ratio with PBS (PH 7.4) was added on smear and incubated at 370 for 30-45 min in humidified dark chamber. The slides were then washed with PBS thrice for 5 min each which will washed away the unbound antibody and then washed with distilled water to stop the reaction. After washing, slides were air-dried and mounted in 10-20 \% glycerine buffer ( $\mathrm{pH} 7.4$ ) or used Dako Faramount Aqueous Mounting Medium (ready to use) and covered with coverslip. The slides were examined under fluorescent microscope at 20X. The presence of apple green fluorescence was considered as positive results (Figure 2).

\section{Data analysis}

The data management and analysis were done in Microsoft excel 2010 (Redmond Microsoft, USA) and Stata version 14.0 (Stata Corp, USA). A two by two table (Table 1) was constructed to calculate the test sensitivity, specificity, positive predictive value, negative predictive of the rapid Ag detection test comparing to the gold standard FAT.

where, true positive (TP) is the number of samples with true-positive results as determined by the gold standard FAT and was also tested positive by Rapid Ag test. The false negative (FN) is the number of samples tested positive to rabies virus by FAT but tested negative by Rapid Ag test. The true negative (TN) is the number of samples with true-negative results determined both by FAT and Rapid Ag test while the false positive (FP) is the number of samples tested negative by FAT but tested positive by rapid Ag test. From Table 1, sensitivity was calculated with the formula $[T P /(T P+F N)] \times 100$ while the specificity was defined as $[T N /(T N+F P)] \times 100$. The positive predictive value $(P P V)$ was calculated as $[T P /(T P+F P)]$ $\times 100$ and the negative predictive value $(N P V)$ as $[T N /(T N+F N)] \times 100$. The confidence intervals for the sensitivity and specificity were calculated by using the exact binomial distribution. The test agreement between FAT and rapid Ag test was calculated using Kappa test. Kappa measures whether a test correctly predicts an outcome. The Kappa value was interpreted as: below 0.0 poor agreement; $0.00-0.20$ slight agreement; $0.21-0.40$ fair agreement; $0.41-0.60$ moderate agreement; $0.61-0.80$ substantial agreement and $0.81-1.00$ almost perfect agreement [11].

\section{Results}

The comparison of the diagnostic methods was carried out using different animal species brain tissue samples. Among 179 samples examined in this study, there was concordance in results by the rapid test and FAT in 115 positive samples and 54 negative samples. Test result were discordant in 10 samples which were positive by FAT, but negative (false negative) by rapid test kit. Thus, the rapid antigen detection test kit had sensitivity of $92 \%$ (95\% Cl: 85.9 - 95.6) and specificity of $100 \%$ (95\% Cl: $93.4-$ 100 ) when compared with FAT. The positive and negative predicative values were found to be $100 \%$ (95\% Cl: 96.7 - 100) and $84 \%$ (95\% Cl: 73.6 - 91.3), respectively. Overall there was $94.41 \%$ (95\% Cl: $90-96.9)$ test agreement (almost perfect agreement) between rapid test and FAT (Kappa value $=0.874$ ). The test discrepancies were observed with dog and cattle samples. The rapid test kit tested 45 dog sample 
negative when compared to only 37 samples being tested negative by FAT (i,e, 8 sample were tested as false negative by rapid test). Similarly, rapid test kit tested 9 cattle sample negative when compared to only 7 samples tested negative by FAT (i,e, 2 sample were tested as false negative by rapid test) (Table 5).

\section{Discussion}

The use of rapid diagnostic tests in the field can greatly enhance disease surveillance activities, especially in resource poor settings. In the present study we have evaluated a commercially available rabies rapid kit (BioNoteC) for its fit-for-purpose in comparison with FAT, the gold standard test for rabies. We first screened all suspected rabies brain samples using the rapid test in the field and then re-confirmed by the FAT. The results showed that the rapid test is highly specific and sensitive, but also encountered some false negative results. The rapid kit's inability to correctly detect 10 FAT-positive samples (10 out of 179 (5.6\%) were false negatives) in our study could have been due to the low viral load in the samples which could not be detected by the rapid kit as compared with the FAT since the rapid kit has low analytical sensitivity to be able to detect only about 102.0LD50/0.03 $\mathrm{ml}$ in mice in both brain and saliva samples. Therefore, confirmatory tests using FAT and molecular tests are usually performed especially in cases of inconclusive and negative results. This could also have been partly due to human factors such as lack of experience of the technicians performing the tests. Other studies have reported diagnostic sensitivities either higher (95 to 100\%) [12-18] or lower (88.3 to 91.7\%) [19-21]. However, one study [22] detected poor sensitivity of the existing commercially available rapid kits when compared with FAT and molecular test. Most of the studies conducted elsewhere had evaluated the rapid test with FAT and other tests in the laboratory setting where the samples were collected from the field and shipped to the laboratory for analysis, and possibly the tests were performed by trained technicians for all the samples thus enhancing the repeatability and reproducibility of the test, thereby reducing the chances of false negatives. In contrast, in our study, the disease investigation team comprising of the veterinarian and the laboratory technician visited the outbreak area or suspect cases, harvested the fresh brain tissues from carcasses and in most cases performed test in the field (on site of death of animals) providing immediate results to the community or farm owners for control measures. Therefore, variation of rapid test results in our study could be due to the varying capacity of the field veterinarians and technicians who may not have been trained on the use of rapid kits. Although the positive test results indicate positive to rabies virus while the negative test results were interpreted cautiously but were always correlated with the history of bite and clinical signs of rabies. The rapid test results could have been improved if same technician performed all the test, but this was not feasible logistically. Moreover, we did not perform rapid test for the second time at the national laboratory other than FAT to reconfirm the rapid test results submitted from the field to avoid wastage of the limited kits. Although simple and easy, a refresher training on rabies diagnosis and surveillance to the field technicians and veterinarians on rapid test protocol is recommended to enhance test result and enhance rabies surveillance.

Like in other studies, our results highlight the potential value of the rapid test kit (BioNote) for countries with limited diagnostic resources. In the past, lack of point-of-care tests delayed the implementation of rapid response to suspected rabies outbreaks since samples had to be transported for days to the 
national laboratory for confirmation even though the preemptive control measures were implemented based on clinical signs and history. Following the introduction of this rapid kit in Bhutan in 2012, the epidemiologic surveillance and diagnosis of rabies in remote areas have greatly improved. The use of rabies rapid tests complemented with epidemiological findings have enhanced the capacity of the field veterinary centres to respond promptly to any suspected outbreaks of rabies and have also rationalized the use of post exposure prophylaxis in cases of positive test results. However, in the event of negative rapid test results, with strong suspicion for rabies (based on clinical signs and other epidemiological features), samples are invariably referred to national laboratory for confirmation using FAT. The current rabies guidelines [23] recommends the use of PEP for all dog bite cases, especially in the rabies endemic areas, and thus the serious implications arising out of decisions made based on false negatives is thus taken care of. In all circumstances, negative cases are further confirmed using FAT and results are accordingly relayed back to the field units for follow up and shared with human health counterparts too. However, it should be noted that rapid test alone should not be used as a confirmatory test for rabies particularly when the test result is negative.

The main advantage of the rapid test kit is that it is inexpensive (cost about USD 7 per kit), rapid and easy to use in the field or in laboratory setting without the need for a microscope, electricity and yield results within 5-10 minutes. Therefore, at least a field test such as this one is better than no diagnostic method at all. Our data, along with those from many previous studies, also support the use of rapid test for rabies surveillance in regions where FAT diagnostic facilities cannot be practically implemented. In resourceconstrained countries such as Bhutan, such rapid kits have greatly enhanced the possibility of providing rapid response to suspected rabies cases and reduced further spread of rabies both in animals and humans. As Bhutan is close to eliminating dog-mediated human rabies, enhanced and sensitive surveillance is a prerequisite to rapidly detect and eliminate the infection and the virus. The use of rapid testing to diagnose rabies in the field contributes to enhanced surveillance across Bhutan; supports judicious use of PEP; and ultimately potentiates the achievement of elimination of dog-mediated rabies.

\section{Declarations}

\section{Ethics approval and consent to participate}

This study was done as part of a routine disease surveillance and an emergency response activity to investigate and control rabies outbreak by the rapid response team. The sampling and test were conducted as per the standard operating procedures and no ethical approval was necessary to analyse this data.

\section{Funding}

This study was done as part of a routine disease surveillance and an emergency response activity to investigate and control rabies outbreak by the rapid response team and was funded by the Royal Government of Bhutan. The funding body has no role in the design of the study and data collection, analysis, interpretation and writing of the manuscript. The rapid test kits and FAT conjugates were 
provided by the World Health Organization Regional Office for South-East Asia, New Delhi, India and the OIE Regional Representation for Asia, Tokyo, Japan.

Availability of data and materials: The dataset analyzed in this study is available upon request from the corresponding author.

\section{Authors' contributions}

TT conceptualized and designed the study; KL, PBR, DT, TT and RBG received field sample and conducted FAT in the laboratory; PG, JN, TW, SL, TR, SJ, CD, SR, LL, KW, LL, PW, TG, KJ, and investigated the outbreak, harvested brain tissues from the carcasses, performed rapid test in the field work and shipped samples to the national laboratory; KD provided administrative support; TT analyzed the data and written the manuscript. All authors read and approved the final manuscript

\section{Competing interests}

The authors declare that they have no competing interests.

\section{Consent for publication}

Not applicable

\section{Acknowledgements}

The authors acknowledge the support provided by laboratory technicians and livestock staffs during outbreak investigation and sampling of the brain tissues samples. We would also like to thank World Health Organization Regional Office for South-East Asia, New Delhi, India and the OIE Regional Representation office in Tokyo for providing Anigen rabies Ag test (BioNote Inc, Seoul, Korea) and rabies conjugates for FAT.

\section{Authors' information}

${ }^{1}$ National Centre for Animal Health, Department of Livestock, Serbithang, Thimphu

${ }^{2}$ Dzongkhag Veterinary Hospital, Trashigang

${ }^{3}$ Regional Livestock Development Centre, Kanglung, Trashigang

${ }^{4}$ City Veterinary Hospital and Satellite Veterinary Laboratory, Phuentsholing

${ }^{5}$ Regional Livestock Development Centre, Tshimasham, Chukha

${ }^{6}$ Satellite Veterinary Laboratory, Deothang, Samdrup Jongkhar

${ }^{7}$ Dzongkhag Veterinary Hospital, Samtse 
${ }^{8}$ Regional Livestock Development Centre, Zhemgang

${ }^{9}$ Satellite Veterinary Laboratory, Gelephu, Sarpang

${ }^{10}$ Satellite Veterinary Laboratory, Nganlam, Pemagatshel

\section{References}

1. Hampson $\mathrm{K}$, et al. (global alliance for rabies control Partners for Rabies). Estimating the global burden of endemic canine rabies. PLoS Negl Trop Dis. 2015;9(4):e0003709. doi:10.1371/journal.pntd.0003709.

2. Tenzin, Dhand NK, Dorjee J, Ward MP. Re-emergence of rabies in dogs and other domestic animals in eastern Bhutan, 2005-2007. Epidemiol Infect. 2010;139:220-5.

3. Tenzin T, Namgyal J, Letho S. Community-based survey during rabies outbreaks in Rangjung town, Trashigang, eastern Bhutan, 2016. BMC Infect Dis. 2017; 17(1), 281.

4. Tenzin T, Rinzin K, Penjor K, Dukpa K, Jamtsho RK, Phuentshoek Y, Mahat H, Choden K, Gyeltshen K, Darnal JB. Rabies Prevention and Control Program in Bhutan: Self-assessment using SARE Tool. Bhutan J Ani Sciences 2019; 3(1):68-78.

5. Tenzin, Dhand NK, Gyeltshen R, Firestone S, Zangmo C, Dema C, Ward M P. Dog bites in humans and estimating human rabies mortality in rabies endemic areas of Bhutan. PLoS neglected tropical diseases, 2011; 5(11), e1391-e1391.

6. Tenzin T, Wangchuk S, Dorji T, McKenzie J, Jolly P. Dog bites and Human Rabies: Epidemiological Analysis of post exposure prophylaxis in Bhutan (2009-2012), Bhutan J Ani Sc. 2017,1(1): 57-64

7. Penjor K, Tenzin T, Jamtsho RK. Determinants of health seeking behavior of animal bite victims in rabies endemic South Bhutan: a community-based contact-tracing survey. BMC Public Health, 2019;19(1), 237. doi:10.1186/s12889-019-6559-x

8. WHO, OIE, FAO, \& GARC. Zero by 30: The Global Strategic Plan to end human deaths from dogmediated rabies by 2030. Geneva; 2018. Retrieved from https://rabiesalliance.org/resource/zero-30global-strategic-plan-end-human-deaths-dog-mediated-rabies-2030.

9. OIE (2019). Manual of diagnostic test and vaccine for terrestrial animals 2019. Pp 570-612. https://www.oie.int/standard-setting/terrestrial-manual/access-online/

10. Ahmed K, Wimalaratne O, Dahal N, Khawplod P, Nanayakkara S, Rinzin K, Perera D, Karunanayake D, Matsumoto T, Nishizono A. Evaluation of a Monoclonal Antibody-Based Rapid Immunochromatographic Test for Direct Detection of Rabies Virus in the Brain of Humans and Animals. Am J Trop Med Hyg. 2012;86(4):736-740. 
11. Landis JR and Koch GG. The measurement of observer agreement for categorical data. Biometrics 1977; 33:159-174.

12. Yang DK, Shin EK, Oh YI, Lee KW, Lee CS, Kim SY, Lee JA, Song JY. Comparison of four diagnostic methods for detecting rabies viruses circulating in Korea. J. Vet. Sci. 2012; 13(1), 43-48

13. Lechenne M, Naïssengar K, Lepelletier A, Alfaroukh IO, Bourhy H, Zinsstag J, Dachuex L. Validation of a Rapid Rabies Diagnostic Tool for Field Surveillance in Developing Countries. PLoS Negl Trop Dis. 2016; 10(10): e0005010.

14. Voehl KM and Saturday GA. Evaluation of a rapid immunodiagnostic rabies field surveillance test on samples collected from military operations in Africa, Europe, and the Middle East. US Army Med Dep J. 2014; 27-32.

15. Yale G, GibsonD, Mani RS, Harsha PK, Costa NC, Corfmat J, Otter I, Otter N, Handel IG, Bronsvoort BM, Mellanby RJ, Desai S, Naik V, Gamble L, Mazeto S. Evaluation of an Immunochromatographic Assay as a Canine Rabies Surveillance Tool in Goa, India. Viruses, 2019; 11(7), 649; https://doi.org/10.3390/v11070649

16. Moore MC; Davis RD, Lilley S, Hanlon CA. Evaluation of a Rapid Immunoassay Detection Kit in comparison to the Gold Standard Direct Fluorescent Antibody test for the diagnosis of rabies. AAVLD Annual Conference Proceedings, Minneapolis, MN November 2010.

17. Mshelbwala PP, Abdullahi SU, Maikai BV, Onyiche ET, Ogunkoya AB. Evaluation of Two Rapid Diagnostic Tests for Rabies Diagnosis under Field and Laboratory Conditions in Nigeria. J Vaccines Vaccine 2015; 6: 272. doi:10.4172/2157-7560.1000272

18. Certoma A, Lunt RA, Vosloo W, Smith I, Colling A, Williams DT, Tran T, Blacksell SD. Assessment of a Rabies Virus Rapid Diagnostic Test for the Detection of Australian Bat Lyssavirus. Trop. Med. Infect. Dis. 2018; 3, 109; doi:10.3390/tropicalmed3040109

19. Kang BK, Oh J, Lee C, Park BK, Park Y, Hong K, et al. Evaluation of a rapid immunodiagnostic test kit for rabies virus. J Virol Methods. 2007;145(1):30-36.

20. Servat A, Picard-Meyer E, Robardet E, Muzniece Z, Must K, Cliquet F. Evaluation of a Rapid Immunochromatographic Diagnostic Test for the detection of rabies from brain material of European mammals. Biologicals.2012; 40(1):61-66.

21. Sharma P, Singh CK, Narang D. Comparison of immunochromatographic diagnostic test with Hheminested Reverse transcriptase polymerase chain reaction for detection of rabies virus from brain samples of various species. Vet. World, 2015; 8, 135-138

22. Eggerbauer E, DeBenedictis P, Hoffmann B, Mettenleiter T.C, Schlottau K, Sabeta C.T, Freuling C.M and Muller T. Evaluation of six commercially available rapid immunochromatographic tests for the 
diagnosis of rabies in brain material. PLoS Negl Trop Dis. 2016; 23; 10(6):e0004776.

23. Ministry of Health. National Guideline for Management of Rabies, $2^{\text {nd }}$ edition 2014. Royal Government of Bhutan. Ministry of Health. Department of Public Health. Zoonotic Disease Program, Thimphu, Bhutan; 2014. http://www.health.gov.bt/wp-content/uploads/moh-files/2015/07/NationalGuideline-for-Management-of-Rabies-2nd-Edition-2014.pdf

\section{Tables}

Table 1: Total brain tissue samples collected and used for analysis from different species of animals (20122017).

\begin{tabular}{lccccccl}
\hline \multirow{2}{*}{ Dzongkhag } & \multicolumn{7}{c}{ Species of animals } \\
\cline { 2 - 7 } & Dog & Cattle & Cat & Goat & Horse & Bear & Total (percent) \\
\hline Chukha & 24 & 6 & 4 & 1 & 2 & $37(20.67)$ \\
\hline Haa & 1 & & & & & $1(0.56)$ \\
\hline S/Jongkhar & 8 & 6 & & & & $14(7.86)$ \\
\hline Samtse & 8 & 13 & & & & $21(11.73)$ \\
\hline Sarpang & 9 & 6 & 1 & 2 & & $18(10.06)$ \\
\hline Trashigang & 40 & 6 & 3 & & & & $49(27.37)$ \\
\hline Thimphu & 2 & 1 & 1 & & & 1 & $5(2.79)$ \\
\hline Zhemgang & 21 & 12 & 1 & & & & $34(18.99)$ \\
\hline Total & 113 & 50 & 10 & 3 & 2 & 1 & $179(100)$ \\
\hline
\end{tabular}

Table 2: Year-wise distribution of brain tissue samples tested between 2012-2017 (some of the samples that did not meet the criteria were excluded for analysis).

\begin{tabular}{ll}
\hline Year & Number of samples (percent) \\
\hline 2012 & $6(3.35)$ \\
2013 & $25(13.97)$ \\
2014 & $14(7.82)$ \\
2015 & $9(5.03)$ \\
2016 & $36(20.11)$ \\
2017 & $89(49.72)$ \\
\hline Total & $179(100)$ \\
\hline
\end{tabular}

Table 3: A two by two table for diagnostic test calculation

\begin{tabular}{c|l|l}
\hline Rapid Ag test & \multicolumn{2}{|c}{ FAT } \\
\cline { 2 - 3 } & Positive & Negative \\
\hline Positive & True positive (TP) & False positive (FP) \\
\hline Negative & False negative (FN) & True negative (TN) \\
\hline
\end{tabular}

Table 4: Test comparison between fluorescent antibody test (FAT) and rapid antigen test for rabies. 


\begin{tabular}{llll}
\hline & FAT positive & FAT negative & Total \\
\hline Rapid test positive & 115 & 0 & 115 \\
Rapid test negative & 10 & 54 & 64 \\
\hline Total & 125 & 54 & 179 \\
\hline
\end{tabular}

Note: $\mathrm{Se}=115 / 125=92 ; \mathrm{Sp}=54 / 54=100$;

$\mathrm{PPV}=115 / 115=100 ; \mathrm{NPV}=54 / 64=84.4$

Table 5: Comparison of rabies diagnosis results obtained with FAT and Rapid antigen test kit on 179 species of animals in Bhutan (2012-2017).

\begin{tabular}{llcc}
\hline Samples & Result & FAT & Rapid test \\
\hline Bear $(\mathrm{n}=1)$ & Positive & 0 & 0 \\
& Negative & 1 & 1 \\
\hline Cat $(\mathrm{n}=10)$ & Positive & 2 & 2 \\
& Negative & 8 & 8 \\
\hline Cattle $(\mathrm{n}=50)$ & Positive & 43 & 41 \\
& Negative & 7 & 9 \\
\hline Dog $(\mathrm{n}=113)$ & Positive & 76 & 68 \\
& Negative & 37 & 45 \\
\hline Goat $(\mathrm{n}=3)$ & Positive & 3 & 3 \\
& Negative & 0 & 0 \\
\hline Horse $(\mathrm{n}=2)$ & Positive & 1 & 1 \\
& Negative & 1 & 1 \\
\hline \multirow{2}{*}{ Total $(\mathrm{n}=1 \mathbf{1 7 9})$} & Positive & $\mathbf{1 2 5}$ & $\mathbf{1 1 5}$ \\
& Negative & $\mathbf{5 4}$ & $\mathbf{6 4}$ \\
\hline
\end{tabular}

\section{Figures}

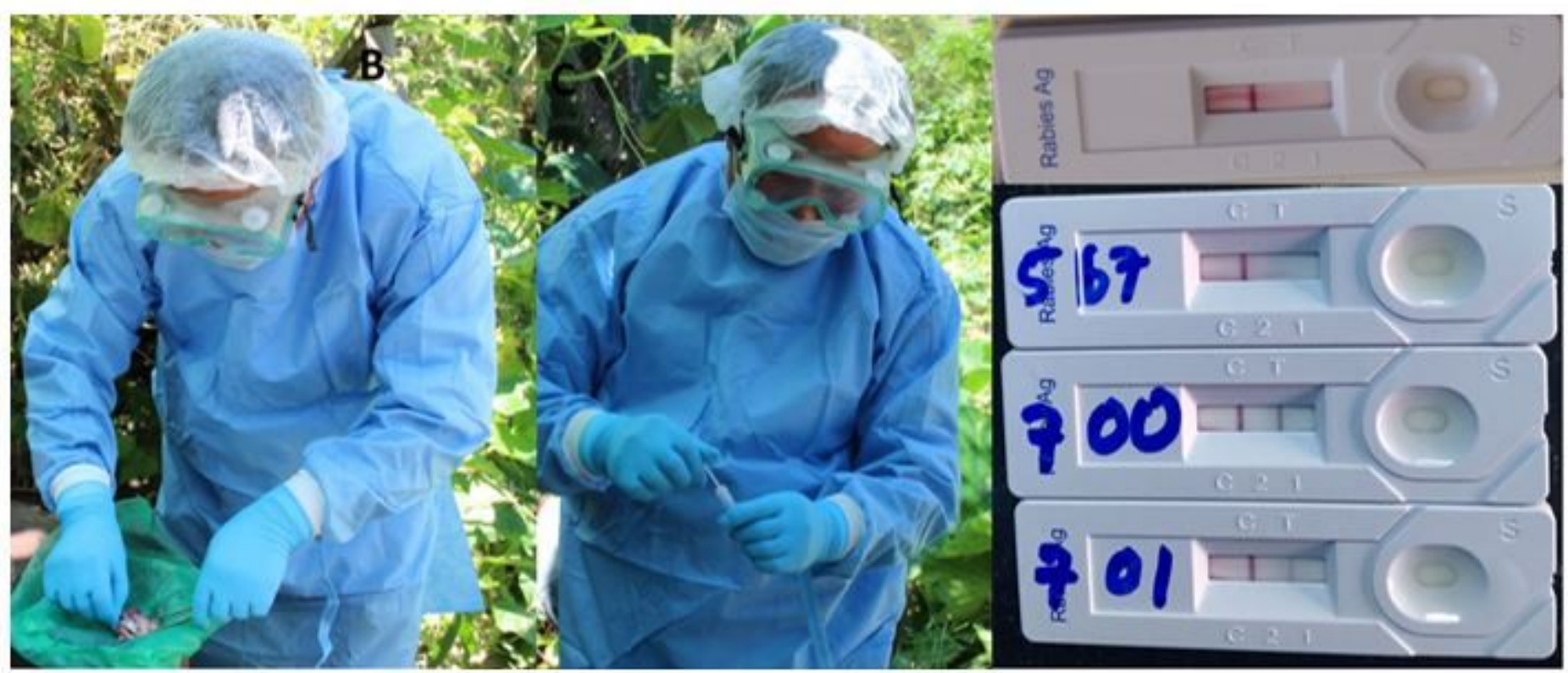

\section{Figure 1}

Harvesting brain tissues from carcass and performing rapid test in the field (on the site of death); rapid test positive to rabies virus showing clear " $\mathrm{C}$ " and " $\mathrm{T}$ " line (bottom 2 test cassette); rapid test negative to 
rabies virus showing only one line (here " $\mathrm{C}$ " line (upper 2 test cassette).
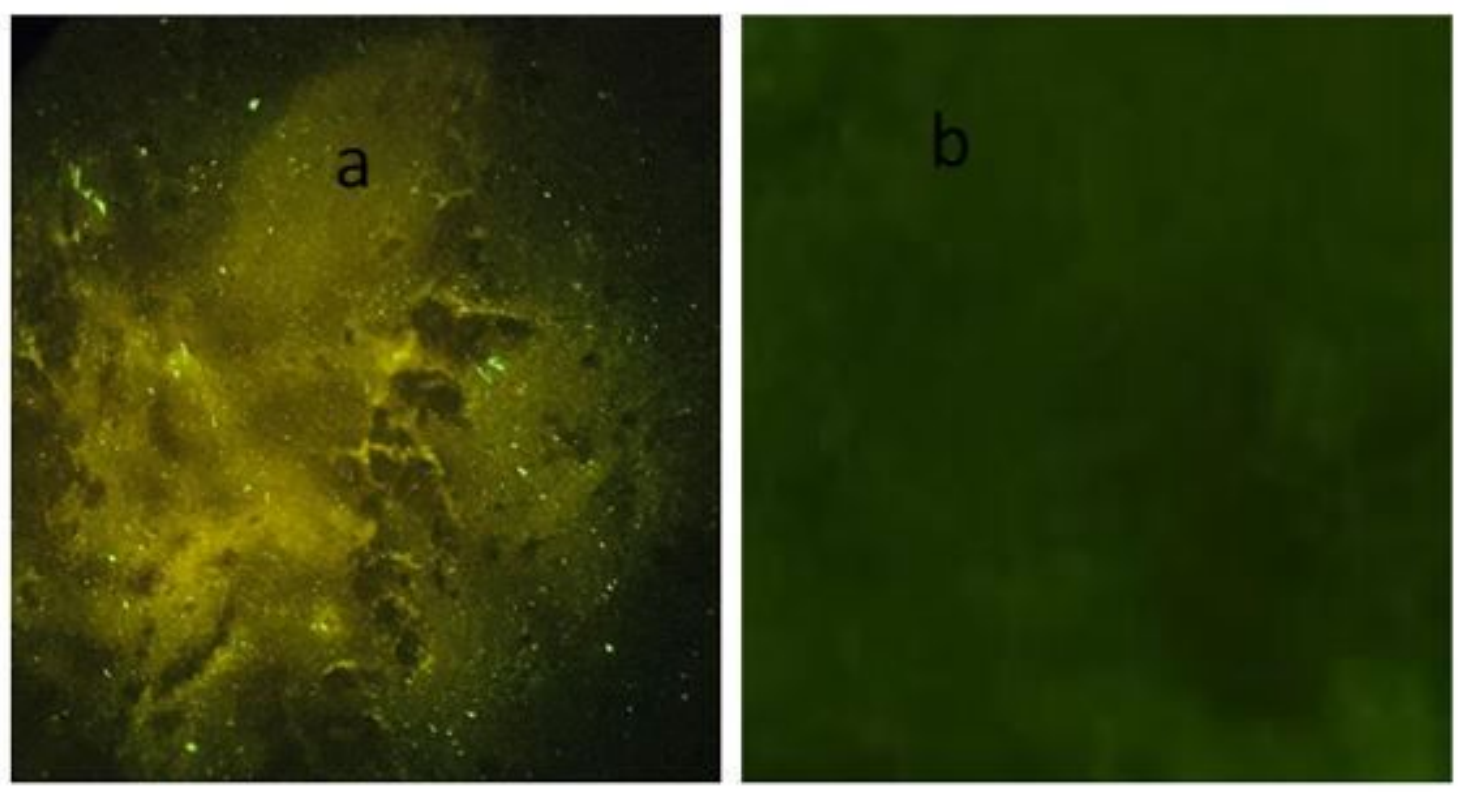

Figure 2

Results of a FAT. Presence of apple green fluorescence - positive to rabies virus (a) and negative to rabies virus (b)

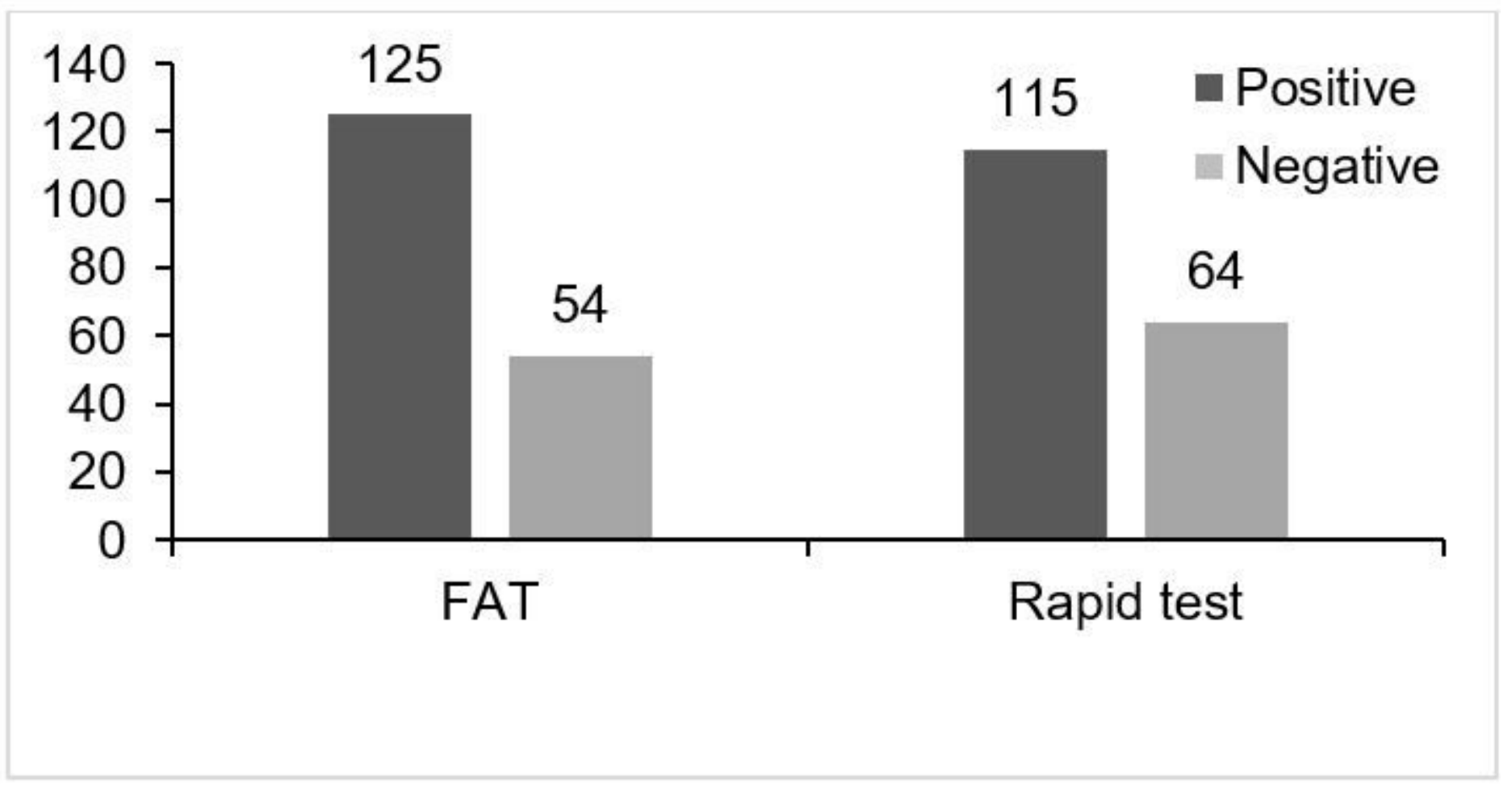

Figure 3

Test comparison between FAT and rapid test 